\title{
Variational finite-difference representation of the kinetic energy operator
}

\section{Citation}

Maragakis, P., José Soler, and Efthimios Kaxiras. 2001. “Variational Finite-Difference Representation of the Kinetic Energy Operator." Physical Review B 64 (19). https:// doi.org/10.1103/physrevb.64.193101.

\section{Permanent link}

http://nrs.harvard.edu/urn-3:HUL.InstRepos:41384081

\section{Terms of Use}

This article was downloaded from Harvard University's DASH repository, and is made available under the terms and conditions applicable to Other Posted Material, as set forth at http:// nrs.harvard.edu/urn-3:HUL.InstRepos:dash.current.terms-of-use\#LAA

\section{Share Your Story}

The Harvard community has made this article openly available.

Please share how this access benefits you. Submit a story. 


\title{
Variational finite-difference representation of the kinetic energy operator
}

\author{
P. Maragakis, ${ }^{1}$ José M. Soler, ${ }^{2,3}$ and Efthimios Kaxiras ${ }^{1,2}$ \\ ${ }^{1}$ Division of Engineering and Applied Sciences, Harvard University, 02138 Cambridge MA \\ ${ }^{2}$ Physics Department, Harvard University, 02138 Cambridge MA \\ ${ }^{3}$ Departamento de Física de la Materia Condensada, \\ Universidad Autónoma de Madrid, 28049 Madrid, Spain
}

\begin{abstract}
A potential disadvantage of real-space-grid electronic structure methods is the lack of a variational principle and the concomitant increase of total energy with grid refinement. We show that the origin of this feature is the systematic underestimation of the kinetic energy by the finite difference representation of the Laplacian operator. We present an alternative representation that provides a rigorous upper bound estimate of the true kinetic energy and we illustrate its properties with a harmonic oscillator potential. For a more realistic application, we study the convergence of the total energy of bulk silicon using a real-space-grid density-functional code and employing both the conventional and the alternative representations of the kinetic energy operator.
\end{abstract}

Electronic structure methods based on finite differences on a real space grid have gained much support in recent years [1, 2] due to their simplicity and versatility. As with plane wave basis sets, their accuracy can be improved easily and systematically. In fact, there exists a rigorous cutoff for the plane waves that can be represented in a given grid, without aliasing, that provides a convenient connection between the two schemes. Soft [3] and ultrasoft [4] pseudopotentials, developed in the plane wave context, can be applied equally well in grid-based methods, resulting in an accurate and efficient evaluation of the potential energy. In contrast with plane waves, the evaluation of the kinetic energy by finite differences is approximate, but it can be significantly improved by using high order representations of the Laplacian operator [5, 6, 7. However, an important difference between finite difference schemes and basis set approaches is the lack of a Rayleigh-Ritz variational principle in the former case. With finite differences, the accuracy of the calculation can also be improved systematically by increasing the grid cutoff (i.e. the grid density). But denser grids generally result in higher energies. This feature, common to all existing real-space-grid approaches to electronic structure calculations, has been discussed frequently in the literature (see for example the discussion of equation (20) in Ref. [2]). It is one of the reasons why it is difficult to develop extrapolation methods and convergence schemes based on minimizing the total energy.

In this paper we show that the origin of the "anti variational" behavior of the total energy in real-space-grid approaches lies in a systematic underestimation of the kinetic energy by the finite-difference representation of the Laplacian operator, independently of the order used. We propose a simple way to construct, for any order, an alternative Laplacian representation that leads to kinetic energies that are higher or equal to the true kinetic energy. The paper is organized as follows. We first briefly present a way to construct the conventional finite difference representation of a Laplacian and show that the spectrum of the resulting operator is lower than the true one. We then apply a variation of this theme to construct a representation which has a spectrum higher than the true one. We compare the convergence of the one-dimensional harmonic oscillator energy using the two representations. Finally we implement the new representation in a real-space electronic structure code and examine the convergence properties of the calculations of bulk Si.

We will consider a three-dimensional (3D) Cartesian coordinate system, in which the Laplacian is the sum of three one-dimensional (1D) operators. For a regular grid in one dimension, a general finite-difference expression for the Laplacian of function $\psi(x)$ at point $x_{i}$ is

$$
\nabla^{2} \psi_{i}=\frac{1}{a^{2}} \sum_{j=-N}^{N} c_{j} \psi_{i+j},
$$

where $\psi_{i} \equiv \psi\left(x_{i}\right)$. The constant $1 / a^{2}$ takes care of the dependence on the grid interval $a$, so that the coefficients $c_{j}$ are independent of it. Thus, we will use $a=1$ for simplicity in what follows.

One way to obtain the Laplacian coefficients $c_{j}$ is through its eigenvalue equation $E(k)=k^{2}$, where $E(k)$ is (except for a constant factor) the kinetic energy of a single plane wave:

$$
E(k)=-\mathrm{e}^{-\mathrm{i} k x} \nabla^{2} \mathrm{e}^{\mathrm{i} k x}=-c_{0}-2 \sum_{j=1}^{N} c_{j} \cos (k j),
$$

where we have used $c_{-j}=c_{j}$ due to the parity of the Laplacian. Notice that $E(k)$ is periodic by construction, with period $2 \pi$, and that its slope is necessarily zero at the grid's Nyquist limit $k= \pm \pi$. Since we have only $N+1$ discrete coefficients to impose $E(k)=k^{2}$ in the continuous range $[-\pi, \pi]$, this can be done only approximately. In an electronic structure calculation, most of the spectral weight is concentrated at low values of $k$. Therefore, a sensible prescription is to require the Laplacian to be accurate around $k=0$, by requiring that the value of $E(k)-k^{2}$ and its $N$ first even derivatives be zero 
at this point. The resulting values of $c_{j}$ are given in Table I of Ref. 月. However, because of its periodic character, and its zero slope at $k=\pi$, the resulting $E(k)$ is a lower bound of the true kinetic energy $k^{2}$, as is shown in Fig. 1. With increasing discretization order $N, E(k)$ approaches $k^{2}$, but the convergence is always from below.

A simple variation of the above theme leads to a representation whose corresponding kinetic energy operator is an upper bound to the true kinetic energy [8]. Instead of fitting $N$ derivatives of $E(k)$ at $k=0$, we fit only the value and the first $N-1$ even derivatives at $k=0$ and, additionally, the value at $k=\pi$. In Table 1 we present the resulting coefficients for orders $N=1-6$.

\begin{tabular}{ccccc}
$N$ & $c_{i}$ & $c_{i+1}$ & $c_{i+2}$ & $c_{i+3}$ \\
\hline 1 & $\frac{-\pi^{2}}{2}$ & $\frac{\pi^{2}}{4}$ & & \\
2 & $-\frac{1}{2}-\frac{3 \pi^{2}}{8}$ & $\frac{\pi^{2}}{4}$ & $\frac{1}{4}-\frac{\pi^{2}}{16}$ & \\
3 & $-\frac{5}{6}-\frac{5 \pi^{2}}{16}$ & $\frac{1}{12}+\frac{15 \pi^{2}}{64}$ & $\frac{5}{12}-\frac{3 \pi^{2}}{32}$ & $-\frac{1}{12}+\frac{\pi^{2}}{64}$ \\
4 & $-\frac{77}{72}-\frac{35 \pi^{2}}{128}$ & $\frac{8}{45}+\frac{7 \pi^{2}}{32}$ & $\frac{23}{45}-\frac{7 \pi^{2}}{64}$ & $-\frac{8}{45}+\frac{\pi^{2}}{32}$ \\
5 & $-\frac{449}{360}-\frac{63 \pi^{2}}{256}$ & $\frac{4}{15}+\frac{105 \pi^{2}}{512}$ & $\frac{59}{105}-\frac{15 \pi^{2}}{128}$ & $-\frac{82}{315}+\frac{45 \pi^{2}}{1024}$ \\
6 & $-\frac{2497}{1800}-\frac{231 \pi^{2}}{1024}$ & $\frac{26}{75}+\frac{99 \pi^{2}}{512}$ & $\frac{493}{840}-\frac{495 \pi^{2}}{4096}-\frac{103}{315}+\frac{55 \pi^{2}}{1024}$ \\
\hline$N$ & $c_{i+4}$ & $c_{i+5}$ & $c_{i+6}$ & \\
\hline 4 & $\frac{17}{720}-\frac{\pi^{2}}{256}$ & & & \\
5 & $\frac{311}{5040}-\frac{5 \pi^{2}}{512}$ & $-\frac{2}{315}+\frac{\pi^{2}}{1024}$ & & \\
6 & $\frac{2647}{25200}-\frac{33 \pi^{2}}{2048}$ & $-\frac{31}{1575}+\frac{3 \pi^{2}}{1024}$ & $\frac{1}{600}-\frac{\pi^{2}}{4096}$
\end{tabular}

TABLE I: Laplacian expansion coefficients leading to upper bound representation of the kinetic energy operator.

In Fig. 11 we show the kinetic energy of a plane wave as a function of its wavevector $k$, in the range $[0, \pi]$. The middle line is the exact kinetic energy, the lower curve is the energy of the conventional Laplacian representation of order $N=6$, and the upper curve is the result of the new Laplacian representation of order 6 . It is clearly seen that the new representation gives an upper bound to the true kinetic energy.

The simplest example that clearly demonstrates the properties of the new Laplacian in action is the 1D harmonic oscillator. We show the gradual convergence of the calculation using the two representations by increasing the number of points used to sample the harmonic oscillator potential $\frac{1}{2} x^{2}$. In Fig. 20 w we the lowest eigenvalue, whose converged value is 0.5 , versus the number of points that sample the interval $[-5,5]$. We use a sixth order representation of the Laplacian for both calculations. The convergence is faster with the conventional representation, because it samples better the low energy part of the spectrum, but the convergence is from below, as expected. In contrast, the new representation converges from above with increasing hamiltonian size, like a basis-set expansion.

Since the 3D Laplacian in Cartesian coordinates is just a sum of three independent 1D Laplacians, its representation in a uniform grid has $6 N+1$ nonzero elements

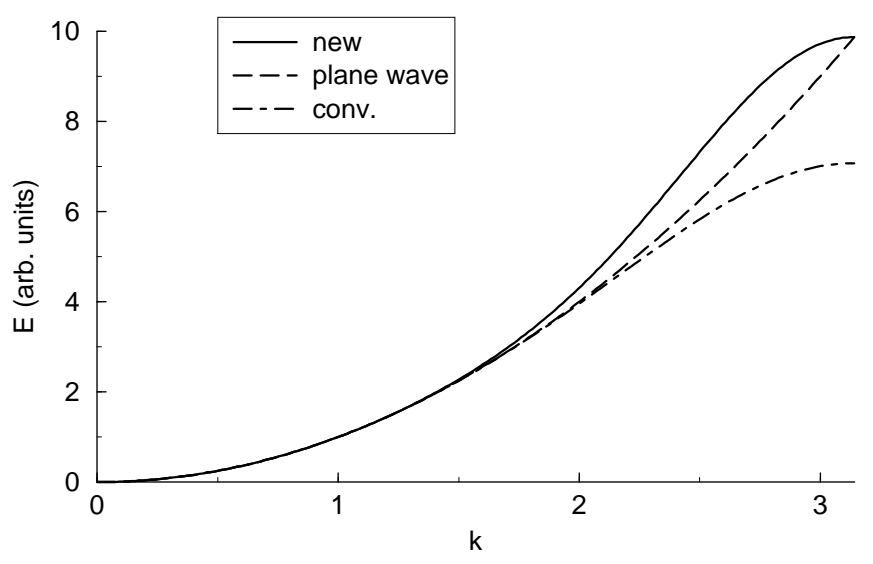

FIG. 1: Middle (dashed) line: exact kinetic energy of a plane wave $E(k)=k^{2}$. Lower (dot-dashed) line: kinetic energy obtained using the conventional finite difference Laplacian representation of order $N=6$. Upper (solid) line: energy of the new Laplacian representation with $N=6$.

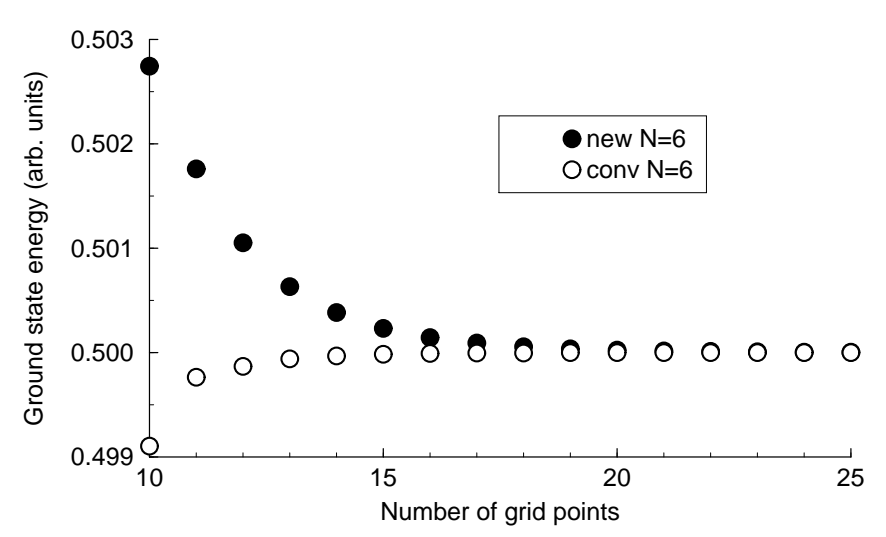

FIG. 2: Convergence of the lowest eigenvalue of the harmonic oscillator with the number of grid points. The filled circles are the results of the upper bound Laplacian representation, the open circles are the results of the conventionalLaplacian.

in a cross orientation, with $3 c_{0}$ at the center and the $N$ elements along the positive and negative sides of the 3 axes. We have implemented both the conventional and the upper bound representation in a real space electronic structure code [9]. We use the new representation in the calculation of the kinetic energy part of the operator, and the conventional representation for the solution of the Poisson problem. The Poisson part of the problem typically converges from above using conventional real-space Laplacians, since the contribution of the plane waves enters with a prefactor of $1 / G^{2}$, where $G$ is the wavevector of the plane wave. For the same reason one can argue that the Poisson problem needs a lower discretization in its solution, since the high energy components are damped by $1 / G^{2}$.

In Fig. 3 we present the convergence characteristics 


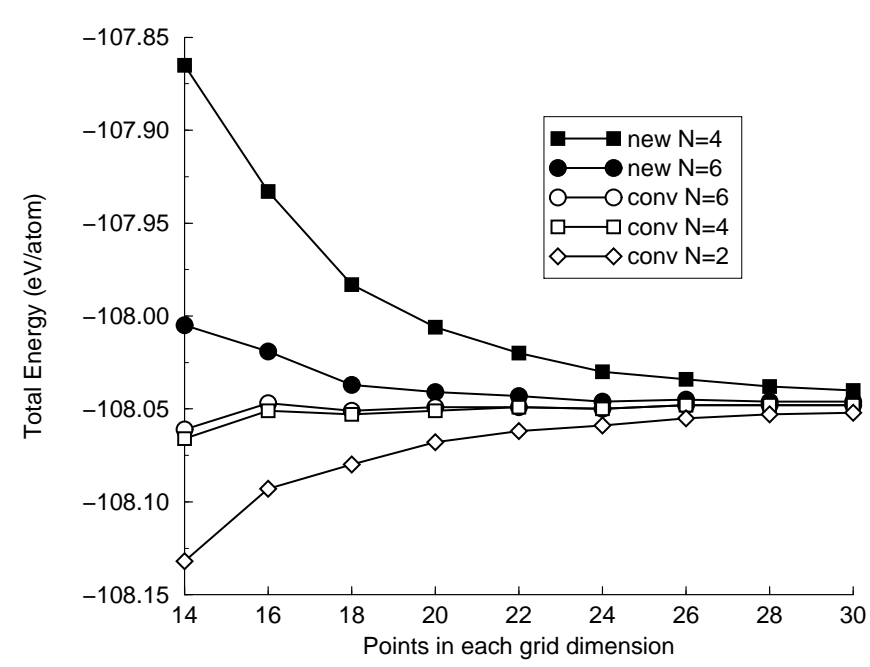

FIG. 3: Convergence of the total energy calculations for Silicon with respect to the number of grid points in each dimension. The curves, from top to bottom correspond to calculations using the new kinetic energy formula (filled symbols) with orders 4 (squares), 6 (circles), and then the conventional formula (open symbols) of order 6 (circles), 4 (squares), and 2 (diamonds). The lines are only used as a guide to the eye.

of a total energy calculation for bulk $\mathrm{Si}$, using normconserving pseudopotentials [10]. An 8 atom diamond lattice cubic unit cell is used with a $6 \times 6 \times 6 k$-point grid in the Monkhorst-Pack scheme 11. The lattice constant is fixed at 10.264 a.u. and the real-space grid discretization is gradually increased from 14 to 30 points in each dimension: this corresponds to an effective plane-wave energy cutoff varying from 18 to 84 Ryd. For simplicity, we use the same order for the Laplacian in the kinetic and Hartree energies. The lowest curve (left pointing triangles) shows the results of the second order Laplacian that is used in HARES [7]. The total energy converges from below, implying that the major source of error comes from the kinetic energy operator. The two curves immediately above it (up-pointing triangles and rhombuses) show the total energy convergence using the conventional formulas of order 4 and 6 . The energy converges from below and quickly reaches its correct value. This is partially due to a cancellation of errors between the kinetic and Hartree energies, but the convergence is not necessarily monotonic as is manifest at 16 and 18 grid points per dimension. The uppermost curve (circles) shows the results using the new Laplacian of order 4 for the kinetic energy, and the conventional Laplacian for the Hartree energy. The total energy convergence is slower, similar to the second order conventional representation, because there is no error cancellation, since both the kinetic and
Hartree energies converge from above. Also, fewer terms are used to approximate the low- $k$ region, while fixing the Laplacian value at $k=\pi$ distorts considerably the kinetic energy eigenspectrum of Fig. 11 at low order expansions. Finally, the curve immediately below it (squares) shows the total energy convergence for the new representation of order 6 , which has a very satisfactory numerical converge from above.

In summary, we derived a new finite difference representation of the Laplacian that is guaranteed to give an upper bound value for the kinetic energy operator. We demonstrated this basic property of the 1D Laplacian through the harmonic oscillator potential and implemented and tested the $3 \mathrm{D}$ version in a real-space electronic structure code. We find that for sufficiently high order of the Laplacian, which for the case of bulk Si is 6 , the new form exhibits very satisfactory numerical convergence of the total energy, while establishing the variational character of the real space method.

PM acknowledges the hospitality of Dr. L. A. A. Nikolopoulos at the Institute for Electronic Structure and Lasers, Heraklion, Greece, in July 2000, and the support of the European Commission through TMR grant number ERB FMGE CT950051 (the TRACS Programme at EPCC)". JMS acknowledges support from Fundación Areces and from Spain's MEC grant BFM2000-1312.

[1] J. Bernholc, Phys. Today 52(9), 30 (1999).

[2] T. L. Beck, Rev. Mod. Phys. 72(4), 1041 (2000).

[3] N. Troullier and J. L. Martins, Phys. Rev. B 43, 1993 (1991).

[4] D. Vanderbilt, Phys. Rev. B 41, R7892 (1990).

[5] J. R. Chelikowsky, N. Troullier, K. Wu, and Y. Saad, Phys. Rev. B 50(16), 11355 (1994).

[6] J. R. Chelikowsky, N. Troullier, and Y. Saad, Phys. Rev. Lett. 72(8), 1240 (1994).

[7] N. A. Modine, G. Zumbach, and E. Kaxiras, Phys. Rev. B 55, 10289 (1997).

[8] (????), another possibility, not explored here, would be to fix some of the coefficients by a least square fit. Although the variational character would not be guaranteed, it might well result in practice.

[9] U. V. Waghmare, H. Kim, I. J. Park, N. Modine, P. Maragakis, and E. Kaxiras, HARES: an efficient method for first-principles electronic structure calculations of complex systems (2001), to appear in Comp. Phys. Comm., arXiv: cond-mat/0006183.

[10] G. B. Bachelet, D. R. Hamann, and M. Schlüter, Phys. Rev. B 26, 4199 (1982).

[11] H. J. Monkhorst and J. D. Pack, Phys. Rev. B 13, 5188 (1976). 